\title{
Gauss Symbol and Unified Theory of Gravity and Electromagnetic Field in Reissner-Nordstrom and Kerr-Newman Solution
}

\author{
Sangwha-Yi* \\ Department of Math, Taejon University 300-716, South Korea
}

*Corresponding Author: Sangwha-Yi, Department of Math, Taejon University 300-716, South Korea

\begin{abstract}
Solutions of unified theory equations of gravity and electromagnetism satisfy Einstein-Maxwell equation. Hence, solutions of the unified theory is Reissner-Nordstrom solution in vacuum. We found in revised Einstein gravity tensor equation, the condition is satisfied by 2-order contravariant metric tensor two times product and Gauss symbol.
\end{abstract}

Keywords: General relativity theory, Unified Theory; 2-order contravariant metric tensor two times $p$ roduct; Gauss Symbol

PACS Number: 04,04.90. $+e, 41.12$

\section{INTRODUCTION}

This theory's aim is that we discover the revised Einstein gravity equation had Reissner-Nordstrom solution and Kerr-Newman solution in vacuum.

First, the revised Einstein gravity equation is in recent my paper,

$$
R_{\mu \nu}-\frac{1}{2} g_{\mu v} R+\Lambda g_{\mu v} I\left(g^{\theta \theta}\right)^{2}=-\frac{8 \pi G}{c^{4}} T_{\mu v}
$$

In this time,

$$
\Lambda=k \frac{G Q^{2}}{C^{4}}, \quad I=\left(\begin{array}{cccc}
1 & 0 & 0 & 0 \\
0 & 1 & 0 & 0 \\
0 & 0 & -1 & 0 \\
0 & 0 & 0 & -1
\end{array}\right)
$$

If we think new revised Einstein gravity field equation using Gauss symbol for replacing the constant matrix $\operatorname{Iin} \mathrm{Eq}(1)-(2)$.

$$
\begin{aligned}
& \Lambda=k \frac{G Q^{2}}{C^{4}}, \\
& I=\left(\begin{array}{cccc}
1 & 0 & 0 & 0 \\
0 & 1 & 0 & 0 \\
0 & 0 & -1 & 0 \\
0 & 0 & 0 & -1
\end{array}\right) \text { is replaced by }(-1)^{\left[\frac{\mu+v}{4}\right]}
\end{aligned}
$$

In this time, Gauss symbol $[x]$ is the greatest integer that is less than or equal to $x$. For example, $[3.141592]=3,[2.71828]=2,[-0.802]=-1, \ldots$ 
Hence, new term is in Kerr-Newman solution,

$$
\begin{aligned}
& (\mu, v)=(0,0) \rightarrow(-1)^{\left[\frac{\mu+v}{4}\right]}=1,(\mu, v)=(1,1) \rightarrow(-1)^{\left[\frac{\mu+v}{4}\right]}=1 \\
& (\mu, v)=(0,3) \rightarrow(-1)^{\left[\frac{\mu+v}{4}\right]}=1(\mu, v)=(3,0) \rightarrow(-1)^{\left[\frac{\mu+v}{4}\right]}=1 \\
& (\mu, v)=(2,2) \rightarrow(-1)^{\left[\frac{\mu+v}{4}\right]}=-1,(\mu, v)=(3,3) \rightarrow(-1)^{\left[\frac{\mu+v}{4}\right]}=-1
\end{aligned}
$$

Hence, new symmetric revised Einstein gravity field Equation is

$$
R_{\mu \nu}-\frac{1}{2} g_{\mu \nu} R+\Lambda g_{\mu \nu}(-1)^{\left[\frac{\mu+v}{4}\right]}\left(g^{\theta \theta}\right)^{2}=-\frac{8 \pi G}{C^{4}} T_{\mu \nu}
$$

We take the covariant differential operator,

$$
\begin{aligned}
& \left(R_{\mu \nu}-\frac{1}{2} g_{\mu \nu} R\right)_{; \mu}+\Lambda g_{\mu \nu}(-1)^{\left[\frac{\mu+v}{4}\right]} 2 g^{\theta \theta} g_{; \mu}^{\theta \theta}=-\frac{8 \pi G}{C^{4}} T_{\mu v ; \mu}=0 \\
& \left(R_{\mu \nu}-\frac{1}{2} g_{\mu \nu} R\right)_{\nu}+\Lambda g_{\mu \nu}(-1)^{\left[\frac{\mu+v}{4}\right]} 2 g^{\theta \theta} g_{\nu}^{\theta \theta}=-\frac{8 \pi G}{C^{4}} T_{\mu \nu \nu}=0
\end{aligned}
$$

In this time, in Kerr-Newman solution[4]

$$
\begin{aligned}
& g_{\theta \theta}=1 / g^{\theta \theta}=\rho^{2}=r^{2}+a^{2} \cos ^{2} \theta \\
& g_{; \rho}^{\theta \theta}=\frac{\partial g^{\theta \theta}}{\partial x^{\rho}}+2 \Gamma_{\sigma \rho}^{\theta} g^{\sigma \theta}=\frac{\partial g^{\theta \theta}}{\partial r}+2 \Gamma_{\theta r}^{\theta} g^{\theta \theta} \\
& =\frac{\partial}{\partial r}\left(\frac{1}{\rho^{2}}\right)+2 \cdot \frac{r}{\rho^{2}} \cdot \frac{1}{\rho^{2}}=-2 \frac{1}{\rho^{3}} \cdot \frac{r}{\rho}+\frac{2 r}{\rho^{4}}=0 \\
& g_{i \rho}^{\theta \theta}=\frac{\partial g^{\theta \theta}}{\partial x^{\rho}}+2 \Gamma_{\sigma \rho}^{\theta} g^{\sigma \theta}=\frac{\partial g^{\theta \theta}}{\partial \theta}+2 \Gamma_{\theta \theta}^{\theta} g^{\theta \theta} \\
& =\frac{\partial}{\partial \theta}\left(\frac{1}{\rho^{2}}\right)-2 \cdot \frac{1}{\rho^{2}} \cdot \frac{1}{2} \rho \frac{2 a^{2}}{\rho} \cos \theta \sin \theta \cdot \frac{1}{\rho^{2}} \\
& =-2 \cdot \frac{1}{\rho^{3}} \cdot-\frac{2 a^{2} \cos \theta \sin \theta}{\rho}-\frac{4 a^{2}}{\rho^{4}} \cos \theta \sin \theta=0
\end{aligned}
$$

If $g_{i \rho}^{\theta \theta}=V_{\rho}$, the vector transformation is

$$
0=V_{\rho}=\frac{\partial x^{\alpha}}{\partial x^{\rho}} V_{\alpha}^{\prime}, \quad V_{\alpha}^{\prime}=0
$$

Therefore, if the coordinate is not the spherical coordinate, the covariant differential of $g^{\theta \theta}=\frac{1}{\rho^{2}}$ is still zero in the changed coordinate

\section{THE NEW REVISED EINSTEIN GRAVITY EQUATION AND REISSNER-NORDSTROM SOLUTION} In this theory, $\mathrm{Eq}(5)$ can change the following equation.

$$
R_{\mu \nu}=-\frac{8 \pi G}{C^{4}}\left(\Gamma_{\mu \nu}-\frac{1}{2} g_{\mu \nu} T_{\lambda}^{\lambda}\right)+\Lambda g_{\mu \nu}(-1)^{\left[\frac{\mu+\nu}{4}\right]}\left(g^{\theta \theta}\right)^{2}
$$


In this time, in vacuum, $\mathrm{Eq}(5)$ is in Reissner-Nodstrom solution.

$$
R_{\mu \nu}=\Lambda g_{\mu \nu}(-1)^{\left[\frac{\mu+v}{4}\right]}\left(g^{\theta \theta}\right)^{2}=\Lambda g_{\mu \nu}(-1)^{\left[\frac{\mu+v}{4}\right]} \frac{1}{r^{4}}
$$

Reissner-Nordstrom solution of Einstein-Maxwell equation is

$$
\begin{aligned}
& g_{00}=-1+\frac{2 G M}{r C^{2}}-\frac{k G Q^{2}}{r^{2} C^{4}}, g_{11}=1 /\left(1-\frac{2 G M}{r C^{2}}+\frac{k G Q^{2}}{r^{2} C^{4}}\right) \\
& g_{22}=r^{2}, g_{33}=r^{2} \sin ^{2} \theta
\end{aligned}
$$

The proper time of spherical coordinates is

$$
\begin{aligned}
& d \tau^{2}=A(t, r) d t^{2}-\frac{1}{c^{2}}\left[B(t, r) d r^{2}+r^{2} d \theta^{2}+r^{2} \sin ^{2} \theta d \varphi^{2}\right] \\
& (\mu, v)=(0,0) \rightarrow(-1)^{\left[\frac{\mu+v}{4}\right]}=1,(\mu, v)=(1,1) \rightarrow(-1)^{\left[\frac{\mu+v}{4}\right]}=1 \\
& (\mu, v)=(2,2) \rightarrow(-1)^{\left[\frac{\mu+v}{4}\right]}=-1,(\mu, v)=(3,3) \rightarrow(-1)^{\left[\frac{\mu+v}{4}\right]}=-1
\end{aligned}
$$

If we use $\mathrm{Eq}(11), \mathrm{Eq}(13)$, we obtain the Ricci-tensor equations.[3]

$$
\begin{aligned}
& R_{\text {tt }}=-\frac{A^{\prime \prime}}{2 B}+\frac{A^{\prime} B^{\prime}}{4 B^{2}}-\frac{A^{\prime}}{B r}+\frac{A^{2}}{4 A B}+\frac{\ddot{B}}{2 B}-\frac{\dot{B}^{2}}{4 B^{2}}-\frac{\dot{A} \dot{B}}{4 A B}=-\Lambda A \frac{1}{r^{4}} \\
& R_{r r}=\frac{A^{\prime \prime}}{2 A}-\frac{A^{2}}{4 A^{2}}-\frac{A^{\prime} B^{\prime}}{4 A B}-\frac{B^{\prime}}{B r}-\frac{\ddot{B}}{2 A}+\frac{\dot{A} \dot{B}}{4 A^{2}}+\frac{\dot{B}^{2}}{4 A B}=\Lambda B \frac{1}{r^{4}} \\
& R_{\theta \theta}=-1+\frac{1}{B}-\frac{n B^{\prime}}{2 B^{2}}+\frac{n A^{\prime}}{2 A B}=-\Lambda r^{2} \frac{1}{r^{4}}=-\Lambda \frac{1}{r^{2}} \\
& R_{\varphi \varphi}=R_{\theta \theta} \sin ^{2} \theta \\
& R_{t r}=-\frac{\dot{B}}{B r}=0 \\
& R_{t \theta}=R_{t \varphi}=R_{r \theta}=R_{r \varphi}=R_{\theta \varphi}=0
\end{aligned}
$$

In this time, $\quad=\frac{\partial}{\partial r} \quad, \cdot=\frac{1}{c} \frac{\partial}{\partial t}$

If we calculate,

$$
\frac{R_{t t}}{A}+\frac{R_{\underline{r}}}{B}=-\frac{1}{B r}\left(\frac{A}{A}+\frac{B^{\prime}}{B}\right)=-\frac{(A B)^{\prime}}{r A B^{2}}=0
$$

Hence, we obtain this result.

$$
A=\frac{1}{B}
$$

If $\operatorname{Eq}(21)$ inserts $\mathrm{Eq}(16)$,

$$
R_{\theta \theta}=-1+\frac{1}{B}-\frac{\not B B^{\prime}}{2 B^{2}}+\frac{\not A A^{\prime}}{2 A B}=-1+\left(\frac{r}{B}\right)^{\prime}=-\Lambda \frac{1}{r^{2}}
$$


If we solve $\mathrm{Eq}(22)$,

$$
\begin{aligned}
& \frac{r}{B}=r+C+\frac{\Lambda}{r} \\
& \rightarrow A=\frac{1}{B}=1-\frac{2 G M}{r C^{2}}+\frac{k G Q^{2}}{r^{2} C^{4}}, \Lambda=k \frac{G Q^{2}}{C^{4}}, C=-\frac{2 G M}{C^{2}}
\end{aligned}
$$

Therefore, in vacuum, the spherical solution of the revised Einstein gravity equation is ReissnerNordstrom solution.

$$
d \tau^{2}=\left(1-\frac{2 G M}{r C^{2}}+\frac{k G Q^{2}}{r^{2} C^{4}}\right) d t^{2}-\frac{1}{c^{2}}\left[\frac{d r^{2}}{\left(1-\frac{2 G M}{r C^{2}}+\frac{k G Q^{2}}{r^{2} C^{4}}\right)}+r^{2} d \theta^{2}+r^{2} \sin ^{2} \theta d \varphi^{2}\right]
$$

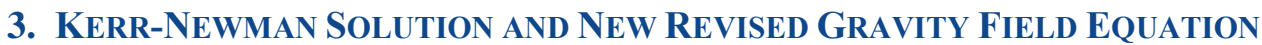

In this theory, $\mathrm{Eq}(5)$ can change the following equation.

$$
R_{\mu \nu}=-\frac{8 \pi G}{C^{4}}\left(F_{\mu \nu}-\frac{1}{2} g_{\mu \nu} T_{\lambda}^{\lambda}\right)+\Lambda(-1)^{\left[\frac{\mu+v}{4}\right]} g_{\mu \nu}\left(g^{\theta \theta}\right)^{2}
$$

In this time, in vacuum, $\mathrm{Eq}(25)$ is in Kerr-Newman solution,

$$
R_{\mu \nu}=\Lambda g_{\mu \nu}(-1)^{\left[\frac{\mu+v}{4}\right]}\left(g^{\theta \theta}\right)^{2}=\Lambda g_{\mu v}(-1)^{\left[\frac{\mu+v}{4}\right]} \frac{1}{\rho^{4}}
$$

We think Kerr-Newman equation and solution.

Kerr-Newman solution is

$$
\begin{aligned}
& d s^{2}=g_{00} c^{2} d t^{2}+2 g_{03} c d t d \phi+g_{11} d r^{2}+g_{22} d \theta^{2}+g_{33} d \phi^{2} \\
& (\mu, v)=(0,0) \rightarrow(-1)^{\left[\frac{\mu+v}{4}\right]}=1,(\mu, v)=\left((, 1) \rightarrow(-1)^{\left[\frac{\mu+v}{4}\right]}=1\right. \\
& (\mu, v)=(0,3) \rightarrow(-1)^{\left[\frac{\mu+v}{4}\right]}=1(\mu, v)=(3,0) \rightarrow(-1)^{\left[\frac{\mu+v}{4}\right]}=1 \\
& (\mu, v)=(2,2) \rightarrow(-1)^{\left[\frac{\mu+v}{4}\right]}=-1,(\mu, v)=(3,3) \rightarrow(-1)^{\left[\frac{\mu+v}{4}\right]}=-1 \\
& \rho^{2}=r^{2}+a^{2} \cos ^{2} \theta
\end{aligned}
$$

Hence, Kerr-Newman equation is[5]

$$
\begin{aligned}
& R_{t t}=\Lambda g_{00} \frac{1}{\rho^{4}} \\
& R_{r x}=\Lambda g_{11} \frac{1}{\rho^{4}} \\
& R_{\theta \theta}=-\Lambda g_{22} \frac{1}{\rho^{4}}=-\Lambda \frac{1}{\rho^{2}} \\
& R_{\phi \phi}=-\Lambda g_{33} \frac{1}{\rho^{4}}
\end{aligned}
$$




$$
\begin{aligned}
& R_{t \phi}=R_{\phi t}=\Lambda g_{03} \frac{1}{\rho^{4}}=\Lambda g_{30} \frac{1}{\rho^{4}} \\
& R_{\phi t}=R_{t \phi}=\Lambda g_{03} \frac{1}{\rho^{4}}=\Lambda g_{30} \frac{1}{\rho^{4}}
\end{aligned}
$$

Otherwise $R_{\mu v}=0$

In this time, for proving $\mathrm{Eq}(28)-\mathrm{Eq}(32)$, Maxwell Equation of Einstein-Maxwell equation is

$$
\begin{aligned}
& -\Lambda g_{\mu \nu}(-1)^{\left[\frac{\mu+v}{4}\right]}\left(g^{\theta \theta}\right)^{2}=R_{\mu \nu}-\frac{1}{2} g_{\mu \nu} R=-\frac{8 \pi G}{C^{4}} T_{\mu \nu} \\
& =-\frac{2 G}{C^{5}}\left(F_{\mu \rho} F_{v}^{\rho}-\frac{1}{4} g_{\mu \nu} F_{\rho \sigma} F^{\rho \sigma}\right) \\
& =-\frac{2 G}{C^{5}}\left(g_{\mu \nu} F_{\nu \rho} F^{v \rho}-\frac{1}{4} g_{\mu \nu} F_{\rho \sigma} F^{\rho \sigma}\right)
\end{aligned}
$$

In spherical coordinates, if we calculate $\mathrm{Eq}(33)$ of $g_{03}, g_{30}[4]$

$$
\begin{aligned}
& -\frac{2 G}{C^{5}}\left(g_{\mu \nu} F_{\nu \rho} F^{\nu \rho}-\frac{1}{4} g_{\mu \nu} F_{\rho \sigma} F^{\rho \sigma}\right) \\
& =-\frac{2 G}{C^{5}}\left(g_{03} F_{32} F^{32}-\frac{1}{4} g_{03}\left(2 B^{2}-2 E^{2}\right)\right) \\
& =-\frac{2 G}{C^{5}}\left(g_{03} B^{2}-\frac{1}{2} g_{03}\left(B^{2}-E^{2}\right)\right)=-\frac{G}{C^{5}}\left(B^{2}+E^{2}\right) g_{03}=-k \frac{G}{C^{4}} \frac{Q^{2}}{\rho^{4}} g_{03}=-\Lambda g_{03} \frac{1}{\rho^{4}}=-\Lambda g_{30} \frac{1}{\rho^{4}} \\
& B^{2}+E^{2}=\frac{Q^{2}}{\rho^{4}}
\end{aligned}
$$

According to $\mathrm{Eq}(35)$, in Kerr-Newman solution, the electromagnetic energy-momentum tensor's formula is equal with the new revised Einstein equation's added term of our unified theory. Hence, we know if we solve the revised Einstein's equation, we can obtain Kerr-Newman solution.

\section{CONClusion}

We found the revised Einstein equation of unified theory (the gravity and electromagnetic field).This theory's strong point is 4-dimensional theory. This theory is different from 5-dimensional KaluzaKlein theory. But as the method of describing universe, Einstein normal gravity equation is equal with the revised Einstein equation of the unified theory because the electric charge has to be zero. .

\section{REFERENCES}

[1]S.Yi, "Spherical Solution of Classical Quantum Gravity", International Journal of Advanced Research in Physical Science, 6,8,2019,pp3-6

[2]S.Yi, "Unified Theory of Gravity and Electromagnetic Field", International Journal of Advanced Research in Physical Science, 6,10,2019,pp10-12

[3]S.Yi, "Unified Theory of Gravity and Electromagnetic Field in Reissner-Nodstrom, Kerr-Newman Solution", International Journal of Advanced Research in Physical Science,6,11,2019,pp1-3

[4]S.Yi, "Unification for Gravity and Electromagnetic Field in Kerr-Newman Solution", International Journal of 
Gauss Symbol and Unified Theory of Gravity and Electromagnetic Field in Reissner-Nordstrom and Kerr-Newman Solution

Advanced Research in Physical Science,6,12,2019,pp11-13

[5]S.Yi, "Unified Theory of Gravity and Electromagnetic Field with Problem in Reissner-Nodstrom and KerrNewman Solution”, International Journal of Advanced Research in Physical Science,7,1,2020, pp12-15

[6]S.Weinberg,Gravitation and Cosmology(John wiley \& Sons,Inc,1972)

[7]P.Bergman,Introduction to the Theory of Relativity(Dover Pub. Co.,Inc., New York,1976), Chapter V

[8]C.Misner, K,Thorne and J. Wheeler, Gravitation(W.H.Freedman \& Co.,1973)

[9]S.Hawking and G. Ellis,The Large Scale Structure of Space-Time(Cam-bridge University Press,1973)

[10]R.Adler,M.Bazin and M.Schiffer,Introduction to General Relativity(McGraw-Hill,Inc., 1965)

Citation: Sangwha-Yi, (2020). “Gauss Symbol and Unified Theory of Gravity and Electromagnetic Field in Reissner-Nordstrom and Kerr-Newman Solution ". International Journal of Advanced Research in Physical Science (IJARPS) 7(7), pp.8-13, 2020.

Copyright: (C) 2020 Authors, This is an open-access article distributed under the terms of the Creative Commons Attribution License, which permits unrestricted use, distribution, and reproduction in any medium, provided the original author and source are credited. 